\title{
Simplistic Coulomb Forces in Molecular Dynamics: Comparing the Wolf and Shifted-Force Approximations
}

\author{
J. S. Hansen,* Thomas B. Schrøder, and Jeppe C. Dyre \\ DNRF Centre "Glass and Time”, IMFUFA, Department of Science, Systems and Models, Roskilde University, Postbox 260, DK-4000 \\ Roskilde, Denmark
}

ABSTRACT: In this paper we compare the Wolf method to the shifted forces (SF) method for efficient computer simulation of bulk systems with Coulomb forces, taking results from the Ewald summation and particle mesh Ewald methods as representing the true behavior. We find that for the HansenMcDonald molten salt model the SF approximation overall reproduces the structural and dynamical properties as accurately as does the Wolf method. It is shown that the optimal Wolf damping parameter depends on the property in focus and that neither the potential energy nor the radial distribution function are useful measures for the convergence of the Wolf method to the Ewald summation method. The SF approximation is also tested for the SPC/Fw model of liquid water at room temperature, showing good agreement with both the Wolf and the particle mesh Ewald methods; this confirms previous findings

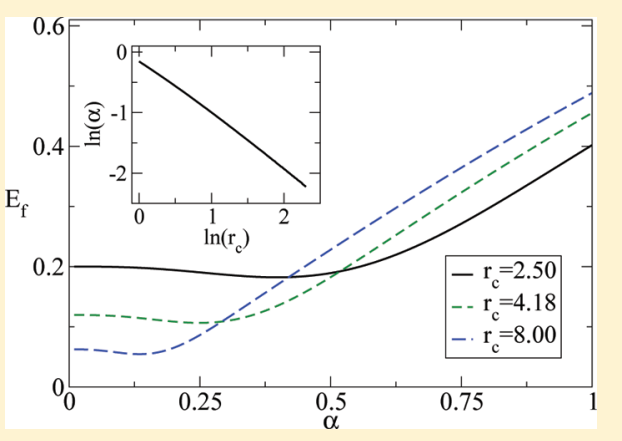
[Fennell, C. J.; Gezelter, J. D. J. Chem. Phys. 2006, 124, 234104]. Besides its conceptual simplicity, the SF approximation implies a speed-up of a factor of 2-3 compared to the Wolf method. We conclude that for the systems studied, whenever the Wolf method gives accurate results, it may be replaced by the simpler and faster SF method.

\section{INTRODUCTION}

Coulomb forces are ubiquitous in nature and must be taken into account in molecular dynamics simulations of polar molecules, ionic liquids, and other charged systems. Because of the long-range nature of the Coulomb interaction this presents a formidable numerical and conceptual challenge which was addressed by Ewald ${ }^{1}$ in the early 1920s. While the Ewald summation method enables a numerical solution to the problem, it requires very long computational time. Since then modified and optimized Ewald-type algorithms have been successfully developed; ${ }^{2}$ these schemes have significantly reduced the computational time for larger systems allowing more complicated phenomena to be studied. In 1999 Wolf et al. $^{3}$ presented an alternative method that allows for even faster simulations of systems with Coulomb forces. This represented a great leap forward; their original paper has been cited more than 250 times and the method is currently applied to, for instance, hydrogen bond switching studies, ${ }^{4}$ in the study of membrane embedded protein systems, ${ }^{5}$ evaluation of transport coefficients in liquid water, ${ }^{6}$ molecular dynamics potentials for dissociative water, ${ }^{7}$ and electrolyte solutions. ${ }^{8}$ In this paper we discuss the further simplification based on a simple shiftedforces cutoff, which is $2-3$ times faster than the Wolf method. We find that whenever the Wolf method adequately approximates the Ewald method, the shifted force method produces comparable results.

In molecular dynamics simulations the force evaluation consumes by far the most computational time. For short-ranged potentials such as the Lennard-Jones function, ${ }^{9}$ it is common to truncate the interaction at a cutoff $r_{\mathcal{c}}$, leading to optimization methods such as inclusion of cell and neighbor lists. ${ }^{10-12}$
Traditionally, the pair potential is simply truncated and some times also shifted such that it is zero at $r_{\mathrm{c}}{ }^{10-12}$ This does not affect the force acting between particles at distances smaller than $r_{\mathrm{c}}$ and if $r_{\mathrm{c}}$ is sufficiently large, the fluid properties are virtually unaffected by this approximation. In fact, it has been shown $^{9,13}$ that keeping merely the short-ranged and purely repulsive part of the van der Waals interaction can account for the fluid structure even near the critical point where correlations are long ranged. The truncated and shifted potential approximation ensures continuity of the potential energy, but can introduce a discontinuity in the force at $r_{\mathrm{c}}$, leading to energy drift for long simulation times. ${ }^{14}$ To overcome this one can instead apply a truncated and shifted force (SF) approximation, ${ }^{10}$ which has superior numerical stability. ${ }^{14}$ Beside the numerical stability, it was recently shown by Toxvaerd and Dyre ${ }^{14}$ that for highly dense fluids the SF method allows for quite small cutoff radius $r_{\mathrm{c}}=1.5 \sigma$ (where $\sigma$ is the atomic diameter) that corresponds to the first local minimum in the radial distribution function. Applying such a low cutoff to the truncated and shifted potential leads to wrong physics and large energy drift. ${ }^{14}$ The SF method decreases the number of interactions significantly and thus the simulation time. The potential corresponding to the SF interaction does, however, not match the original potential for $r<r_{\mathrm{c}}$ from which the SF interaction was derived. Therefore, thermodynamical properties cannot be compared directly, but derived, for example, from perturbation theory. ${ }^{10,15}$

Received: January 23, 2012

Revised: April 10, 2012

Published: April 12, 2012 

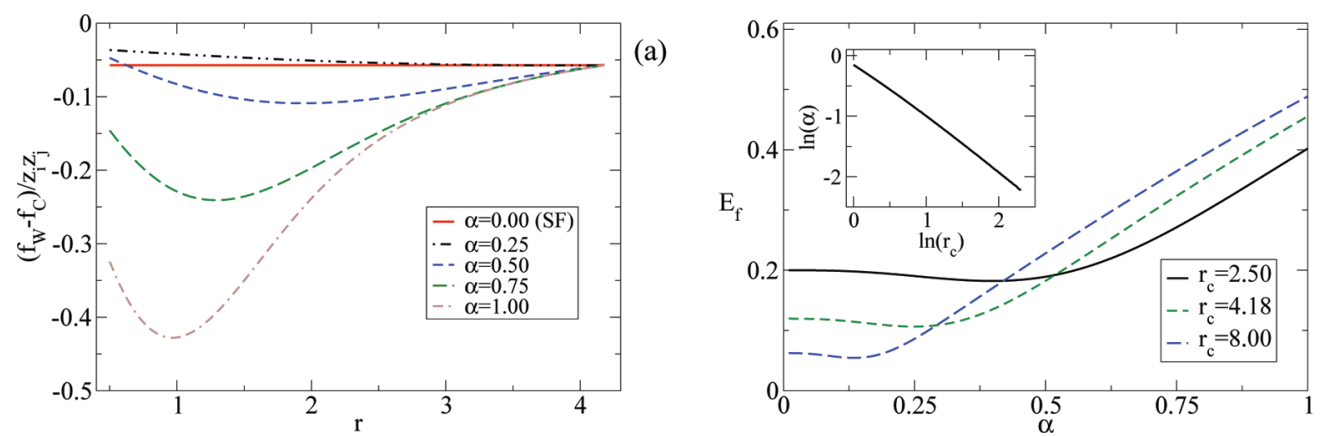

(b)

Figure 1. (a) Difference between the Wolf force, $f_{\mathrm{W}}$, and the Coulomb force, $f_{\mathrm{C}}$, for $\alpha=0.0,0.25,0.5,0.75$, and 1.0 . In all graphs the cutoff is given by $r_{\mathrm{c}}=4.18$. (b) The measure of the difference between the true Coulomb force and the Wolf force, $E_{\mathrm{f}}$, defined in eq 3 plotted as a function of $\alpha$ for three different cutoffs. The inset shows the optimal value of $\alpha$ plotted as a function of $r_{\mathrm{c}}$.

For long-ranged interactions, like the Coulomb interaction, one cannot simply introduce a standard cut and shifted potential. For example, simply truncating and shifting the Coulomb potential produces unnatural fluid structure and spurious dynamics. ${ }^{16}$ Numerous attempts have been made to overcome this problem. For example it has been suggested to use smoothing functions, but this leads in general to poor results. ${ }^{17,18}$ Wolf et al. ${ }^{3}$ cleverly showed that using a simple truncated and shifted Coulomb potential corresponds in practice to summing over interactions in a non-neutral sphere. To compensate for this the authors introduced a neutralizing term into the Coulomb potential, they further showed that faster convergence to the true energy is achieved by applying a damping factor $\alpha$. The Wolf method is computationally much faster than the classical Ewald summation technique and it is today applied to various systems as mentioned above. The choice of the damping factor, $\alpha$, is, like the Ewald damping parameter, ${ }^{1,10}$ somewhat arbitrary, and the optimal value must be found by comparison with either experimental data or results from, e.g., the Ewald method. ${ }^{8,18}$ If the Wolf damping parameter $\alpha$ is zero, the Wolf method reduces to the SF approximation; ${ }^{19}$ see also Denesyuk and Weeks ${ }^{20}$ for a discussion. We note that an SF method for the Coulomb interactions was used in the biochemical simulation community $^{21,22}$ before the work by Wolf et al.

In this paper we apply the Wolf method in molecular dynamics simulations of a simple model of a molten salt and liquid water. In order to find the optimal value of the Wolf damping parameter, $\alpha$, we compare the simulated thermodynamical, dynamical, and structural properties with previously published results ${ }^{23}$ based on the Ewald method. We show that the optimal value of $\alpha$ depends on the property one wishes to calculate and the cutoff distance used. This sets the stage for documenting the main results of this paper: for the systems studied here the SF approximation works as well as the Wolf method, confirming similar findings of Fennell and Gezelter. ${ }^{19}$ Besides being conceptual simpler than the Wolf method, the SF method allows for more than a doubling of the computational speed.

\section{WOLF APPROXIMATION TO THE COULOMB POTENTIAL}

If $r$ is the distance between two particles, the force acting on one particle due to the interaction with the other is $\mathbf{F}(r)=f(r)$ $\mathbf{r} / r$, where $f$ is from this point on denoted the "force" for simplicity and is minus the derivative of the corresponding potential function with respect to $r$ and $\mathbf{r}$ is the vector of separation. For the Wolf method ${ }^{3}$ the force is given by

$$
\begin{aligned}
f_{\mathrm{W}}\left(r ; \alpha, r_{\mathrm{c}}\right)= & z_{i} z_{j}\left[\frac{\operatorname{erfc}(\alpha r)}{r^{2}}-\frac{\operatorname{erfc}\left(\alpha r_{\mathrm{c}}\right)}{r_{\mathrm{c}}^{2}}\right. \\
& \left.+\frac{2 \alpha}{\sqrt{\pi}}\left(\frac{\exp \left(-\alpha^{2} r^{2}\right)}{r}-\frac{\exp \left(-\alpha^{2} r_{\mathrm{c}}{ }^{2}\right)}{r_{\mathrm{c}}}\right)\right]
\end{aligned}
$$

for $r<r_{\mathrm{c}}$ and where $\operatorname{erfc}(x)=1-\operatorname{erf}(x)$ is the complementary error function. Here $z_{i}$ and $z_{j}$ are the charges of the two particles in question, $r_{\mathrm{c}}$ is the cutoff (i.e., $f_{\mathrm{W}}=0$ for $r \geq r_{\mathrm{c}}$ ), and $\alpha$ is the Wolf damping parameter. In the paper by Wolf et al. it is implicitly understood that $\alpha r_{\mathrm{c}}>1$ such that the cutoff only takes effect beyond the range of damping. The damping parameter was introduced in order to ensure faster convergence to the limiting Madelung energy. ${ }^{3}$ Unfortunately, there is no theoretical prediction for the optimal value of $\alpha$, which must be found by comparison with other well-established methods like the Ewald summation method. ${ }^{3,8,18}$ Wolf et al. ${ }^{3}$ and Demontis et al. $^{8}$ have shown via molecular dynamics simulations that the Wolf method reproduces the results obtained by the Ewald summation method for $r_{\mathrm{c}} \geq 5 d_{i j}$, where $d_{i j}$ is the distance between oppositely charged particles in the first coordinate shell. Demontis et al. ${ }^{8}$ also suggested that the optimal damping parameter is given by $\alpha=2 / r_{\mathrm{c}}$ for sufficiently large systems.

From eq 1 it follows that for $\alpha \rightarrow \infty$ one has $f_{\mathrm{W}} \rightarrow 0$, and that for $\alpha \rightarrow 0$ the force reduces to

$$
f_{\mathrm{SF}}\left(r ; r_{\mathrm{c}}\right)=z_{i} z_{j}\left(1 / r^{2}-1 / r_{\mathrm{c}}^{2}\right) \quad \text { for } r<r_{\mathrm{c}}
$$

This is the truncated and shifted force (SF) function. ${ }^{10,14}$

In Figure 1a we plot the difference between the Wolf force, $f_{\mathrm{W}}$, and the corresponding Coulomb force, $f_{\mathrm{C}}=z_{i} z_{j} / r^{2}$, for different damping parameters. Clearly the damping parameter has a nontrivial effect on the force. For $\alpha=0$ the difference is small compared to large values of $\alpha$, suggesting that the SF method, eq 2, gives a good approximation to the Coulomb interaction. From Figure 1a it is seen that an optimal value of $\alpha$ exists that minimizes the difference. One way to identify this optimal value is by minimizing the function

$$
E_{\mathrm{f}}\left(\alpha, r_{\mathrm{c}}\right)=1-\frac{\int_{0}^{r_{\mathrm{c}}} f_{\mathrm{W}}\left(r ; \alpha, r_{\mathrm{c}}\right) \mathrm{d} r}{\int_{0}^{r_{\mathrm{c}}} f_{\mathrm{C}}(r) \mathrm{d} r}
$$

which measures the total relative difference between $f_{\mathrm{W}}(r)$ and $f_{\mathrm{C}}(r)$ such that $E_{\mathrm{f}} \geq 0\left(\right.$ since $f_{\mathrm{W}} \leq f_{\mathrm{C}}$ for all $\left.r\right)$. In Figure $1 \mathrm{~b} E_{\mathrm{f}}$ 
is plotted for three different cutoff distances. The optimal Wolf damping parameter converges to zero as $r_{\mathrm{c}}$ increases, which reflects the simple fact that $f_{\mathrm{W}} \rightarrow f_{\mathrm{C}}$ for $r_{\mathrm{c}} \rightarrow \infty$ and $\alpha \rightarrow 0$. More interestingly, the quantity $E_{\mathrm{f}}$ exhibits very little difference between the optimal value of $\alpha$ and $\alpha=0$. The inset in Figure $1 \mathrm{~b}$ shows that the optimal Wolf parameter determined by the minimum of eq 3 is given roughly by $\alpha \approx 3 /\left(4 r_{c}\right)$. This simple analysis is consistent with the $r_{\mathrm{c}}$ dependence suggested by Demontis et al. ${ }^{8}$ based on molecular dynamics simulations (but they predict a smaller estimate of $\alpha$ by a factor of $3 / 8$ ).

Figure 2 shows an example of the average difference in total force experienced by the ions in a molten salt system; ${ }^{23}$ see

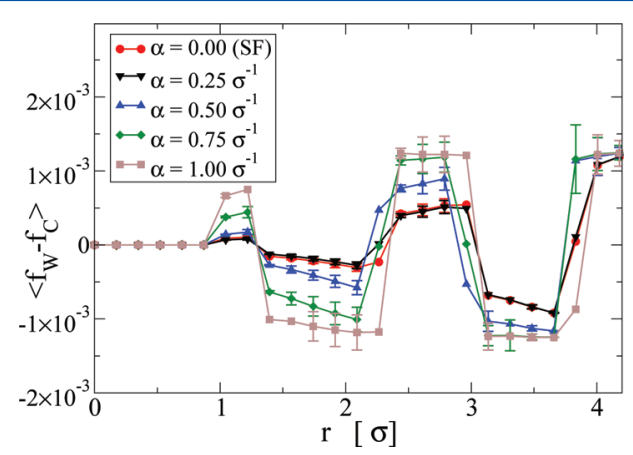

Figure 2. Difference between the Wolf force, $f_{\mathrm{W}}$, and the Coulomb force, $f_{\mathrm{C}}$, for a model molten salt system. ${ }^{23}$ Here $r_{\mathrm{c}}=4.18 \sigma$ and $\alpha=$ $0.0,0.25,0.5,0.75$, and $1.0 \sigma^{-1}$. Note, not all error bars are shown for clarity. The length scale $\sigma$ is approximately one ion diameter.

more details below for this model. In this example the particle positions, i.e., the phase space trajectory, evolve under the influence of the Wolf and van der Waal forces, and at different time intervals the average total force difference, $f_{\mathrm{W}}-f_{\mathrm{C}}$, is computed as a function of distance. It is seen that both the SF and Wolf approximations are in excellent agreement with the true Coloumb force, especially for low values of the damping parameter.

The conclusion from Figures 1 and 2 is that setting $\alpha=0$, i.e., adopting the SF approximation, gives results that are close to those obtained by carefully optimizing $\alpha$. This and the recent work by Toxvaerd and Dyre ${ }^{14}$ motivate the below reported molecular dynamics simulations, which compare the Wolf method to the SF method for other quantities and actual simulations of realistic systems.

\section{RESULTS FOR THE HANSEN-MCDONALD MOLTEN SALT MODEL}

A series of molecular dynamics simulations was performed of a model molten salt proposed by Hansen and McDonald. ${ }^{23}$ Briefly, in this two-component model, the ions are simple spherical particles that interact via a Coulomb potential and a van der Waals type potential, which is given by the inverse power law $\phi(r)=\left(\varepsilon^{2} / n \sigma\right)(\sigma / r)^{n}$, where $n=9, \varepsilon$ defines the energy scale, and $\sigma$ is the usual Lennard-Jones length scale parameter. ${ }^{9}$ We refer the reader to the reference for the full details. In the simulations we applied the Wolf method and varied the cutoff between 2.5 and $8.0 \sigma$. The simulation box used was twice the size of the cutoff whenever $r_{c}>4.18 \sigma$; for smaller cutoffs the box length was fixed to $8.36 \sigma$. The number density for all systems was $\rho=0.368 \sigma^{-3}$, thus the number of ions varied from 216 to 1508 . The results presented below were independent of system size. The temperature $T$ is controlled using a Nosé-Hoover thermostat ${ }^{24,25}$ with $T=0.0177 \varepsilon / k_{B}$. The results are compared to previously published data where the Ewald summation method was used, ${ }^{23}$ which represent the "true" Coulomb interaction.

First, in Figure 3a we compare the total potential energy obtained from the Wolf method $U_{\mathrm{W}}$ for three different cutoff radii and varying damping parameters with the potential energy $U_{\mathrm{E}}$ from the Ewald summation method. We note that $U_{\mathrm{W}}$ is obtained directly from the Wolf potential function ${ }^{3,18}$ corresponding to the force given in eq 1 . It is observed that $U_{\mathrm{W}}$ is within the statistical uncertainty equal to $U_{\mathrm{E}}$ for sufficiently small damping parameters, even for quite small cutoffs. This could lead to the conclusion that the Wolf method accounts correctly for electrostatic interactions for very small cutoff distances. However, if one plots the radial distribution function $g$, Figure $3 \mathrm{~b}$, we see that for $r_{\mathrm{c}}=2.5 \sigma$ the structure differs from the result obtained using the Ewald summation method. This is true for all values of the damping parameter. From Figure $3 \mathrm{~b}$ we also notice that the SF approximation captures the structural properties correctly for $r_{\mathrm{c}}=6 \sigma$, which is the smallest cutoff distance meeting the Wolf et al. ${ }^{3}$ and Demontis et al. ${ }^{8}$ criterion, $r_{\mathrm{c}} \geq 5 d_{i j}$.

We study the radial distribution function dependence of $r_{\mathrm{c}}$ and $\alpha$ by defining the error parameter $E_{\mathrm{g}}$ via

$$
E_{\mathrm{g}}=\frac{\int_{0}^{r_{\mathrm{c}}}\left|g_{\mathrm{W}}(r)-g_{\mathrm{E}}(r)\right| \mathrm{d} r}{\int_{0}^{r_{\mathrm{c}}} g_{\mathrm{E}}(r) \mathrm{d} r}
$$
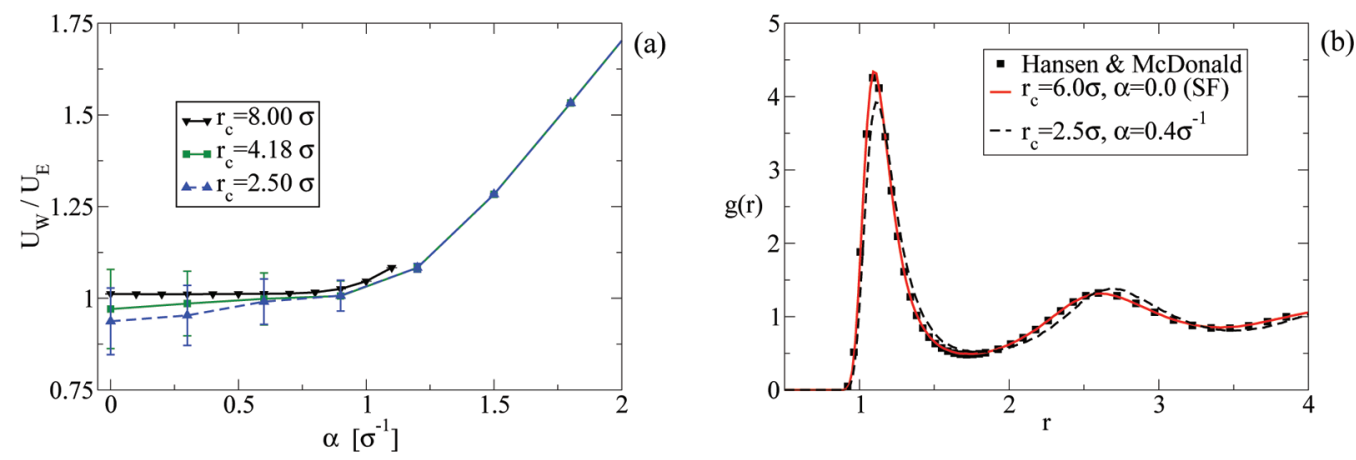

Figure 3. (a) Comparison of the potential energy of the Hansen-McDonald molten salt model for varying damping parameter. Error bars represent the standard error of ten independent runs. $U_{\mathrm{E}}$ is found in Hansen and McDonald. ${ }^{23}$ (b) Radial distribution functions for unlike charged particles (lines) for $\alpha=0$ (the SF approximation) and $r_{\mathrm{c}}=6 \sigma$ and for $\alpha=0.4 \sigma^{-1}$ and $r_{\mathrm{c}}=2.5 \sigma$. The filled black squares are data points of Hansen and McDonald. $^{23}$ 
where $g_{\mathrm{W}}$ is the radial distribution function for unlike charged particles of the Wolf method and $g_{\mathrm{E}}$ is the radial distribution function produced by the Ewald summation method. Similarly, the following error parameter $E_{D}$ quantifies the difference in diffusion constant:

$$
E_{\mathrm{D}}=\frac{D_{\mathrm{W}}}{D_{\mathrm{E}}}-1
$$

where $D_{\mathrm{W}}$ and $D_{\mathrm{E}}$ are the diffusion constants obtained from the Wolf and Ewald methods, respectively. Note that $E_{\mathrm{g}} \geq 0$, whereas $E_{\mathrm{D}}$ can be negative. The "correct" radial distribution function, $g_{\mathrm{E}}$, and diffusion constant, $D_{\mathrm{E}}$, were taken from Hansen and McDonald. ${ }^{23}$ Figure 4 shows the two error

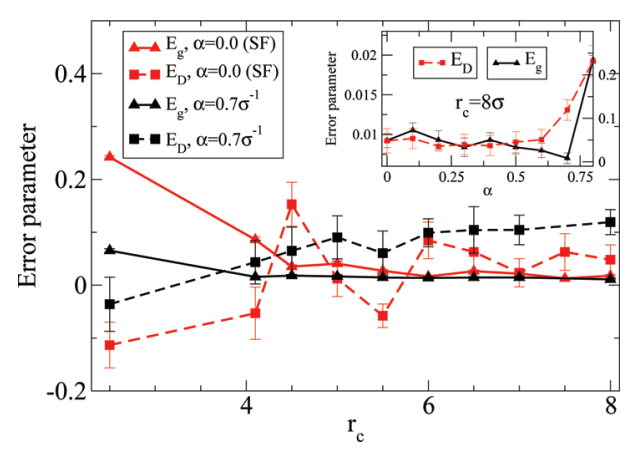

Figure 4. Error parameters as a function of cutoff for different damping parameter for the Hansen and McDonald molten salt system. The inset shows the error parameters for $r_{\mathrm{c}}=8 \sigma$ as functions of $\alpha$.

parameters for different cutoff radii and damping. The damping parameter $\alpha=0.7 \sigma^{-1}$ was chosen because $E_{\mathrm{g}}$ exhibits a minimum for this value for a large range of cutoffs. This is not the case for $E_{\mathrm{D}}$, however, which features a minimum for lower values of the damping parameter, depending on the cutoff (as expected from Figure $1 \mathrm{~b}$ ). This inconsistency is illustrated in the inset in which the error parameters are shown for $r_{\mathrm{c}}=8 \sigma$ as functions of $\alpha$. Obviously, any $\alpha<0.6 \sigma^{-1}$ may be chosen to minimize $E_{\mathrm{D}}$, whereas $E_{\mathrm{g}}$ features a minimum for $\alpha=0.7 \sigma^{-1}$. We note that $r_{\mathrm{c}}=8 \sigma>5 d_{i j}$ so the cutoff radius fulfills the criterion defined by Wolf et al. and Demontis et al.

From Figure 4 it is seen that $E_{\mathrm{g}}$ is relatively large for small cutoffs (as expected), but that it for nonzero damping parameters quickly decreases and reaches almost zero for $r_{\mathrm{c}}>$ $4.0 \sigma^{-1}$. For the SF approximation one needs $r_{\mathrm{c}}>6.0 \sigma$ in order to obtain the same accuracy in the radial distribution function. For large cutoffs the SF approximation results in better diffusion constants than the Wolf method with $\alpha=0.7 \sigma^{-1}$. We could, of course, have optimized $\alpha$ with respect to the diffusion constant (giving $\alpha \cong 0.3 \sigma^{-1}$ for a large range of cutoffs). This, however, would decrease the agreement for the radial distribution function. This fact is highlighted in Table 1, where the error parameters are listed for values of $\alpha$ optimized, respectively, with respect to the diffusion constant and the radial distribution function $\left(r_{\mathrm{c}}=8.0 \sigma\right)$. For comparison we also give the error parameters for the SF approximation. Within the statistical uncertainty there is no difference between the Wolf method using $\alpha=0.3 \sigma^{-1}$ and the SF approximation.

Up to this point we have only discussed the structural and diffusive properties in the long time limit. To compare the short-time dynamics of the two methods we plot the velocity autocorrelation function $C_{v v}(t)$ and the intermediate scattering function in Figure 5. From Figures 3 and 4 it was concluded
Table 1. Error Parameters, $E_{\mathrm{D}}$ and $E_{\mathrm{g}}$, for Different Values of the Damping Parameter ${ }^{a}$

$\begin{array}{lcc}\alpha\left[\sigma^{-1}\right] & E_{\mathrm{D}} & E_{\mathrm{g}} \\ 0.0(\mathrm{SF}) & 0.04 \pm 0.02 & 0.017 \pm 0.002 \\ 0.3 & 0.03 \pm 0.01 & 0.019 \pm 0.002 \\ 0.7 & 0.12 \pm 0.02 & 0.010 \pm 0.001\end{array}$

${ }^{a} \alpha=0.3 \sigma^{-1}$ and $\alpha=0.7 \sigma^{-1}$ correspond to the optimized values with respect to diffusion and radial distribution function, respectively. $\alpha=$ $0.0 \sigma^{-1}$ corresponds to the SF approximation.

that for small $r_{\mathrm{c}}\left(r_{\mathrm{c}} \approx 4.0 \sigma\right)$ and large $\alpha\left(\alpha \approx 0.7 \sigma^{-1}\right)$ both the potential energy and the radial distribution function are in excellent agreement with the Ewald summation method, but in Figure 5 we clearly observe that the short-time dynamics is not correct for this set of parameter values. This shows that the cutoff must be sufficiently large for the Wolf method to correctly account for all the fluid properties, but at such large cutoff the SF approximation may be applied instead since it results in the same accuracy.

\section{RESULTS FOR THE SPC/FW WATER MODEL}

We also tested the SF approximation for liquid water at the state point $(T, \rho)=\left(300 \mathrm{~K}, 998 \mathrm{~kg} \mathrm{~m}^{-3}\right)$ using the flexible single point charge $(\mathrm{SPC} / \mathrm{Fw})$ water model. ${ }^{26}$ In this model the chemical bond and the bending angle are allowed to vibrate around their zero-force values. The model is easy to implement and has been shown to predict many bulk properties better than for example the SPC, SPC/E, and TIP3P models. ${ }^{26,27}$ As it is the case for the molten salt, the Wolf method leads to a potential energy which is in acceptable agreement with the PME method ${ }^{26}$ over a large range of damping parameters. However, as we have discussed above, this measure will not guarantee that the electrostatic interaction are correctly accounted for and yields correct structural and dynamical properties. In Figure 6a we plot the oxygen-oxygen radial distribution function $g_{\mathrm{OO}}$ for the Wolf and SF methods. For comparison, data from $\mathrm{Wu}$ et al. ${ }^{26}$ are shown (filled squares), where the Coulomb interactions were evaluated using the particle-mesh Ewald (PME) method. ${ }^{2}$ The radial distribution function is reproduced reasonably well by both methods. The SF approximation captures the liquid structure at least as well as the Wolf method, except at the first peak which is slightly underestimated and at the cutoff where the SF method induces a small antisymmetric bump in the structure, see figure inset. This bump has also recently been observed by Kale and Herzfeld $^{28}$ for what the authors term "unusual" ionic liquids. Except for the discrepancy around the cutoff, the radial distribution functions for both the SF and the Wolf methods are independent of the cutoff for radii larger than $9 \AA$, the value used by Zahn et al.; ${ }^{18}$ this corresponds to $r_{\mathrm{c}} \approx 5 d_{i j}$ since the oxygen-hydrogen distance is around $1.8 \AA$. In Figure $6 \mathrm{~b}$ the center-of-mass velocity autocorrelation function is plotted for two different cutoffs for both methods. This dynamic property is largely independent of method and cutoff, as is the case for the liquid structure. The same conclusion was reached by Fennell and Gezelter. ${ }^{19}$ For the SF approximation we obtain a diffusion constant of $2.4 \times 10^{-9} \mathrm{~m}^{2} \mathrm{~s}^{-1}$, a shear viscosity of 0.78 $\times 10^{-3} \mathrm{~Pa}$ s and a dielectric constant of $\varepsilon_{\mathrm{r}}=76 \pm 2$. This can be compared with the data where the PME scheme was applied: ${ }^{26}$ $2.32 \times 10^{-9} \mathrm{~m}^{2} \mathrm{~s}^{-1}$ (at $\left.298.15 \mathrm{~K}\right), 0.75 \times 10^{-3} \mathrm{~Pa} \mathrm{~s}$ (at $300.2 \mathrm{~K}$ ) and $\varepsilon_{\mathrm{r}}=79.6$ (at $298.15 \mathrm{~K}$ ). It is also worth mentioning that Zahn et al. ${ }^{18}$ used $\alpha=0.06 \sigma^{-1}$ in their simulations of (rigid) 

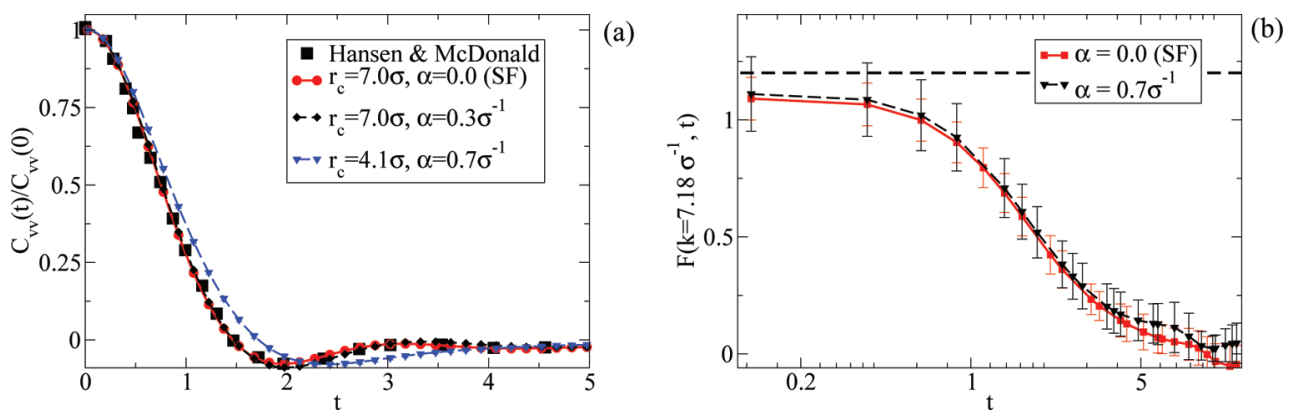

Figure 5. (a) Normalized velocity autocorrelation function for the Wolf and the SF methods. Only the short time data are shown. The error bars are comparable to the size of symbols. (b) Coherent intermediate scattering function for wavelength $k=7.18 \sigma^{-1}$ and $r_{\mathrm{c}}=7.0 \sigma$. The horizontal line is the interpolated value of the static structure factor $S(k)=F(k, 0)$ taken from Hansen and McDonald. ${ }^{23}$ The time $t$ is given in standard reduced molecular dynamics units.
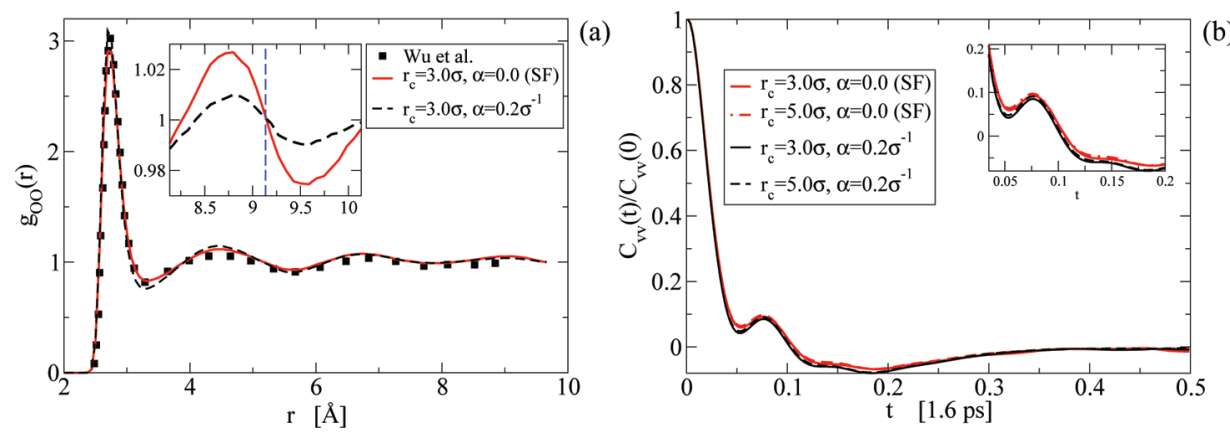

Figure 6. (a) Oxygen-oxygen radial distribution function for the SPC/Fw water model using SF and Wolf methods. The squares represent data taken from Wu et al. ${ }^{26}$ (b) Normalized center-of-mass velocity autocorrelation functions. The inset shows a zoom of the time interval 0.065 to 0.32 ps. In both panels a and b, $\sigma=3.16 \AA$.

SPC/E water, but found that the potential energy was in better agreement with the Ewald method for even lower damping parameters. Again, we find that for sufficiently large cutoff length the SF approximation and Wolf method perform equally well. Finally, we note that the resulting transport properties of $\mathrm{SPC} / \mathrm{Fw}$ water using the SF and Wolf methods are in better agreement with the long ranged PME scheme than is the case for the simple molten salt system. This could be due the fact that the charges are better correlated in the former case.

\section{CONCLUDING REMARKS}

Fennell and Gezelter ${ }^{19}$ carefully analyzed an impressive number of different systems including simple crystals, showing a good agreement between the SF method and the Ewald technique. In their conclusion the authors suggested that the SF approximation can also be used for confined geometries, thereby overcoming the enforced periodicity in the unmodified Ewald method. We agree that the Ewald method can be problematic (even for sufficiently small periodic systems ${ }^{29-32}$ ), but the SF approach (as well as the Wolf method) is an approximation that suppresses the intrinsic long-ranged nature of the Coulomb interactions, leading to an artificially molecular orientation ${ }^{16,33}$ in confinements. For confined fluids alternative methods have recently been advised. ${ }^{20,34,35}$

The Wolf method has one more parameter than the SF approximation and may consequently be optimized to give slightly better agreement with the Ewald summation method. Such an optimization, however, must be carried out separately for each property under study and for each different system. Beside its simplicity (and thus easy-to-code feature), we found that the SF approximation leads to a simulation speed-up of 2-
3 compared to the Wolf method. This estimate is based on two different force evaluation algorithms: (i) a brute force algorithm where all distances between the particles are calculated (an order $N^{2}$ algorithm, where $N$ is the number of particles) and (ii) a nearest-neighbor and cell-list algorithm that scales with the number of particles, i.e., of order $N$. Of course, the actual speed-up depends on the specific problem and the use of optimization techniques, but the calculation of the four terms in eq 1 involves complicated mathematical functions and is bound to consume considerably more computational resources than the simple SF approximation. We wish to stress here that the paper of Wolf et al. was the first to correctly analyze why the SF approximation for Coulomb forces is superior to the standard truncated and shifted potential interaction model.

In conclusion, we find that for bulk molten salt and liquid water systems using the cutoff lengths where the Wolf method correctly accounts for the fluid properties, one may equally well use the simpler and computational more efficient SF approximation.

\section{AUTHOR INFORMATION}

\section{Corresponding Author}

*E-mail: jschmidt@ruc.dk.

\section{Notes}

The authors declare no competing financial interest.

\section{ACKNOWLEDGMENTS}

J.S.H. acknowledges Lundbeckfonden for supporting this work as part of Grant No. R49-A5634. The centre for viscous liquid dynamics "Glass and Time" is sponsored by the Danish National Research Foundation (DNRF). 


\section{REFERENCES}

(1) Ewald, P. Ann. Phys. 1921, 369, 253.

(2) Darden, T.; York, D.; Pedersen, L. J. Chem. Phys. 1993, 98, 10089.

(3) Wolf, D.; Keblinski, P.; Phillpot, S. R.; Eggebrecht, J. J. Chem. Phys. 1999, 110, 8254.

(4) Vreede, J.; Wolf, M. G.; de Leeuw, S. W.; Bolhuis, P. G. J. Phys. Chem. B 2009, 113, 6484.

(5) Kikugawa, G.; Apostolov, R.; Kamiya, N.; Taiji, M.; Himeno, R.; Nakamura, H.; Yonezawa, Y. J. Comput. Chem. 2009, 30, 110.

(6) Hansen, J.; Bruus, H.; Todd, B.; Daivis, P. J. J. Chem. Phys. 2010, 133, 144906.

(7) Mahadevan, T. S.; Garofalini, S. H. J. Chem. Phys. B 2007, 111, 8919.

(8) Demontis, P.; Spanu, S.; Suffritti, G. B. J. Chem. Phys. 2001, 114, 7980.

(9) Mcquarrie, D. A. Statistical Mechanics; Harper and Row: New York, 1976.

(10) Allen, M. P.; Tildesley, D. J. Computer Simulation of Liquids; Clarendon Press: New York, 1989.

(11) Frenkel, D.; Smit, B. Understanding Molecular Simulation; Academic Press: London, 1996.

(12) Rapaport, D. The Art of Molecular Dynamics Simulation; Cambridge University Press: Cambridge, 1995.

(13) Weeks, J. D.; Chandler, D.; Andersen, H. C. J. Chem. Phys. 1971, 54, 5237.

(14) Toxvaerd, S.; Dyre, J. C. J. Chem. Phys. 2011, 134, 081102.

(15) Nicolas, J. J.; Gubbins, K. E.; Streett, W. B.; Tildesley, D. J. Mol. Phys. 1979, 37, 1429.

(16) Feller, S. E.; Pastor, R. W.; Rojnuckarin, A.; Bogusz, S.; Brooks, B. R. J. Phys. Chem. 1996, 100, 17011.

(17) Brooks, C. L.; Pettitt, B. M.; Karplus, M. J. Chem. Phys. 1985, 83, 5897.

(18) Zahn, D.; Schilling, B.; Kast, S. M. J. Phys. Chem. B 2002, 106, 10725.

(19) Fennell, C. J.; Gezelter, J. D. J. Chem. Phys. 2006, 124, 234104.

(20) Denesyuk, N. A.; Weeks, J. D. J. Chem. Phys. 2008, 128, 124109.

(21) Levitt, M.; Hirshberg, M.; Sharon, R.; Daggett, V. Comput. Phys. Commun. 1995, 91, 215.

(22) Beck, D. A. C.; Armen, R. S.; Daggett, V. Biochem. 2005, 44, 609.

(23) Hansen, J. P.; McDonald, I. R. Phys. Rev. A 1975, 11, 2111.

(24) Nosé, S. Mol. Phys. 1984, 52, 255-268.

(25) Hoover, W. G. Phys. Rev. A 1985, 31, 1695-1697.

(26) Wu, Y.; Tepper, H. L.; Voth, G. A. J. Chem. Phys. 2006, 124, 024503.

(27) Raabe, G.; Sadus, R. J. J. Chem. Phys. 2007, 126, 044701.

(28) Kale, S.; Harzfeld, J. J. Chem. Theo. Comp. 2011, 7, 3620.

(29) Karlström, G.; Stenhammer, J.; Linse, P. J. Phys.: Condens. Matter 2008, 20, 494204.

(30) Yonetani, Y. Chem. Phys. Lett. 2005, 406, 49.

(31) Yonetani, Y. J. Chem. Phys. 2006, 124, 204501.

(32) van der Spoel, D.; van Maaren, P. J. J. Chem. Theo. Comp. 2006, $2,1$.

(33) Takahashi, K.; Narumi, T.; Yasouko, K. J. Chem. Phys. 2011, 134, 174112.

(34) Rodgers, J. M.; Weeks, J. D. Proc. Natl. Acad. Sci. U.S.A. 2008, 105, 19136.

(35) Wu, X.; Brooks, B. R. J. Chem. Phys. 2005, 122, 044107. 